\title{
The Effects of Vanadium Substitution on One-dimensional Tunnel Structures of Cryptomelane: Combined TEM and DFT Study
}

Shi-Ze Yang, ${ }^{1,2}$ Killian R. Tallman, ${ }^{3}$ Ping Liu, ${ }^{4}$ Diana M. Lutz, ${ }^{3}$ Bingjie Zhang, ${ }^{3}$ Sung Joo Kim, ${ }^{1,2}$ Lijun $\mathrm{Wu},{ }^{1}$ Amy C. Marschilok, ${ }^{2,3,5}$ Esther S. Takeuchi, ${ }^{2,3,5}$ Kenneth J. Takeuchi, ${ }^{3,5}$ Yimei Zhu ${ }^{1,6}$

${ }^{1}$ Condensed Matter Physics and Materials Science Department, Brookhaven National Laboratory, Upton, New York, United States, ${ }^{2}$ Energy and Photon Sciences Directorate, Brookhaven National Laboratory, Upton, New York, United States, ${ }^{3}$ Department of Chemistry, Stony Brook University, Stony Brook, New York, United States, ${ }^{4}$ Chemistry Division, Brookhaven National Laboratory, Upton, New York, United States, ${ }^{5}$ Department of Materials Science and Chemical Engineering, Stony Brook University, Stony Brook, New York 11794, United States, ${ }^{6}$ Department of Physics and Astronomy, Stony Brook University, Stony Brook, New York, United States

Lithium-ion batteries play a critical role as portable energy storage systems in modern society. The major components of lithium-ion batteries are the cathode, anode, separator, electrolyte and binder. The central scientific problem of these systems that needs to be addressed is how to scientifically advance the different components to improve the rate capability, reduce cost, increase cycle life and achieve safe operation.

Among the candidates, manganese oxides have the special advantage of low-cost.[1,2] Further, the rich structural polymorphs of manganese oxide provide vast opportunities for exploring the effect of material structure on electrochemistry. Tunnel-type (or hollandite) manganese oxide with one-dimensional Li diffusion channels is a very interesting material for studying the fundamental electrochemistry of manganese oxides. Hollandite structures have attracted special attention due to the homogenous tunnel structure and moderate space within the tunnel.

Structural features including particle size, surface defects and volume (bulk) defects can impact the electrochemistry of battery materials. Nanomaterials are beneficial for lithium-ion batteries as they provide a short Li diffusion path length. Both surface structure and the stability of electrode materials are critical to the electrochemistry, as the surface is the interface with the electrolyte and structural stability facilitates the diffusion of Li ions from electrode to electrolyte. Surface coating and doping have been generally adopted to stabilize the surface structure for high voltage battery electrode materials. In addition, surface defects are beneficial for Li diffusion across tunnels. Finally, volume (bulk) defects can be important, as demonstrated by the improved cycling performance in Ni-rich layered cathodes caused by tailoring grain boundaries within the material.

State-of-the-art scanning transmission electron microscopy (STEM) and in-situ TEM techniques are carried in the present study.[3] We substitute $\mathrm{Mn}$ atoms with $\mathrm{V}$ to tune the electrochemistry of cryptomelane $\left(\mathrm{K}_{\mathrm{X}} \mathrm{MnO}_{2}\right)$ from the perspective of its atomic structure. In the pristine nanorod, irregular $2 \times 1$ and $2 \times 3$ boundary tunnels are identified along with the dominant $2 \times 2$ tunnel framework. Upon V substitution, these irregular tunnel sizes are no longer observed while tunnel lengths are reduced. Atomic level electron energy loss spectroscopy (EELS) mapping shows that V dopants occupy the tunnel wall positions, directly substituting Mn atoms as shown in Figure 1a with agrees with the DFT simulation results in Figure 1b-1d very well.[4] 
We carried out in-situ and ex-situ EELS measurements using a nanofactory holder using Li metal with partial oxidation layers to probe the crytomelane samples. Both in-situ and ex-situ results show that valence change of $\mathrm{Mn}$ is much larger than that of $\mathrm{V}$ upon reduction by lithium metal. Consistent with the experimental observations, DFT simulations also reveal the substituted V ions to be in a slightly oxidized state and that upon lithiation $\mathrm{V}$ is reduced only slightly as compared to $\mathrm{Mn}$. In a former theoretical paper,[5] it is predicted that there might be two possible structural evolution mechanism upon lithiation. In our ex-situ study on lithiated samples, we observed the evidence for both evolution mechanisms. Our future work will further clarify the parameters affecting the different structural evolution pathways for the tunnel structures.
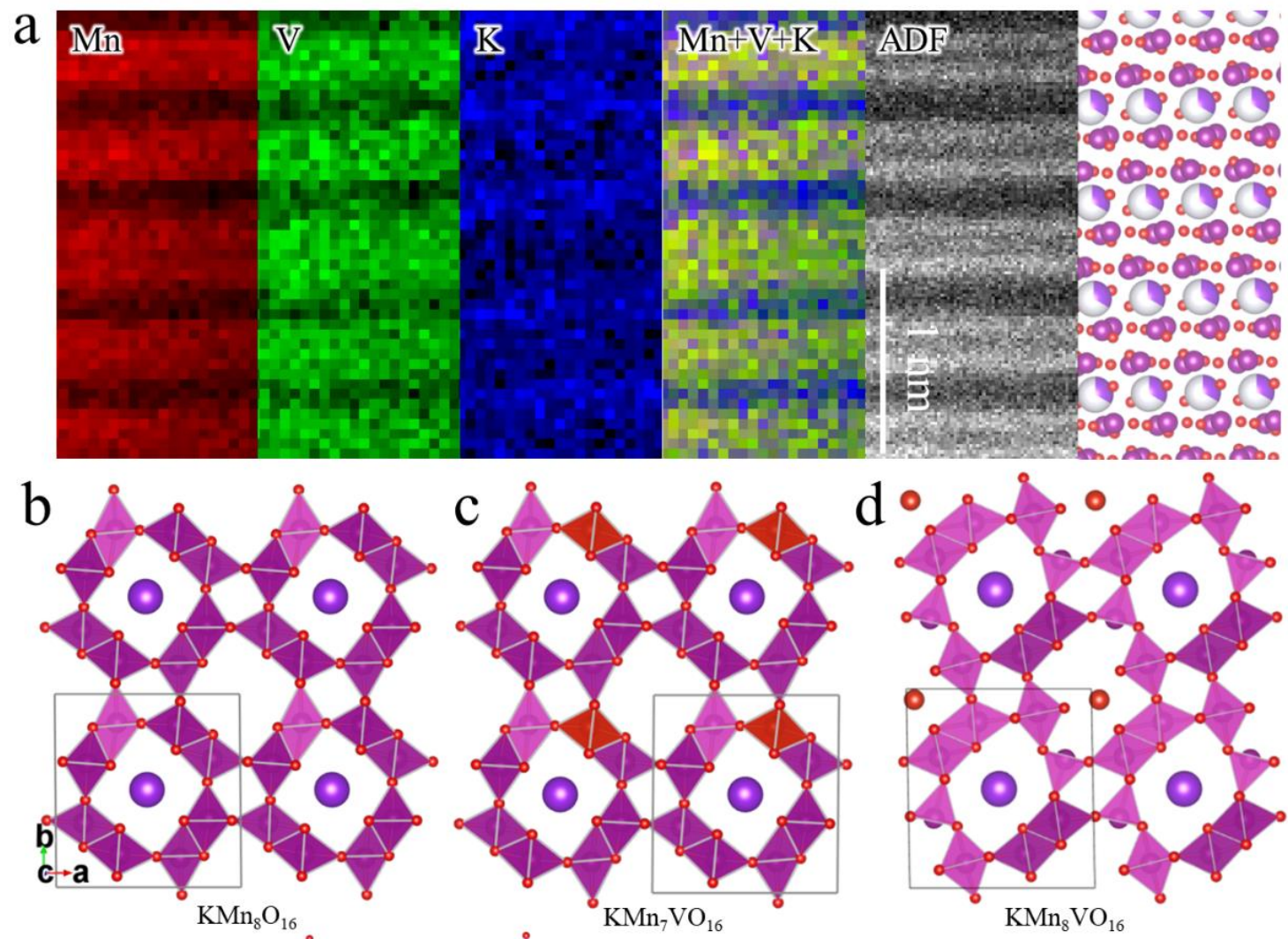

$\mathrm{Mn} \odot \mathrm{Mn}^{+4} \mathrm{O}_{6} \& \mathrm{Mn}^{+4-\mathrm{x}} \mathrm{O}_{6}$ \&
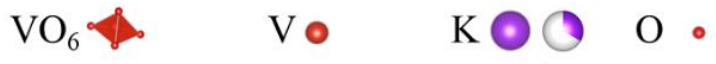

Figure 1. (a) STEM EELS mapping results for the 5\%V-substituted sample (K1.02Mn7.63V0.37O16) showing $\mathrm{Mn}$ (red), V (green) and $\mathrm{K}$ (blue) with composite (Mn-V-K), and related ADF image and structural models shown to the right; (b) Structural model for pristine cryptomelane (KMn8O16), with K+ in the tunnel highlighted as a large ball and the reduced Mn shown in semi-transparent polyhedra; (c) Structural model for V-substituted cryptomelane (KMn7VO16), with dopant in the tunnel wall shown as red polyhedra; (d) Structural model for V-centered cryptomelane (KMn8VO16), with dopant in the tunnel shown as red ball. 


\section{References}

[1] Cai, R., Guo, S., Meng, Q., Yang, S., Xin, H.L., Hu, X., Li, M., Sun, Y., Gao, P., Zhang, S. and Dong, H., 2019. Atomic-level tunnel engineering of todorokite $\mathrm{MnO}_{2}$ for precise evaluation of lithium storage mechanisms by in situ transmission electron microscopy. Nano Energy, 63, p.103840.

[2] Wang, Y., Feng, Z., Yang, S.Z., Gagnon, C., Gariépy, V., Laul, D., Zhu, W., Veillette, R., Trudeau, M.L., Guerfi, A. and Zaghib, K., 2018. Layered oxides-LiNi1/3Co1/3 $\mathrm{Mn}_{1 / 3} \mathrm{O}_{2}$ as anode electrode for symmetric rechargeable lithium-ion batteries. Journal of Power Sources, 378, pp.516-521.

[3] Yang, Shi-Ze, Weiwei Sun, et al. "Direct cation exchange in monolayer $\mathrm{MoS}_{2}$ via recombinationenhanced migration." Physical Review Letters 122, no. 10 (2019): 106101.

[4] Yang, S.Z., Tallman, K.R., Liu, P., Lutz, D.M., Zhang, B., Kim, S.J., Wu, L., Marschilok, A.C., Takeuchi, E.S., Takeuchi, K.J. and Zhu, Y., 2020. The effects of vanadium substitution on onedimensional tunnel structures of cryptomelane: Combined TEM and DFT study. Nano Energy, 71, (2020) p.104571.

[5] A.B. Brady, K.R. Tallman, E.S. Takeuchi, A.C. Marschilok, K.J. Takeuchi, P. Liu, J. Phys. Chem. C 123 (41) (2019) 25042-25051. 